\title{
An improved segmentation method for multi-material beam hardening correction in industrial X-ray computed tomography
}

\author{
Wenchao Cao ${ }^{1}$, Sam Hawker ${ }^{2}$, Gemma Fardell ${ }^{2}$, Ben Price ${ }^{2}$, \\ Wim Dewulf ${ }^{1}$ \\ Department of Mechanical Engineering, KU Leuven, Celestijnenlaan 300, 3001, \\ Leuven, Belgium ${ }^{1}$ \\ X-Tek Systems Ltd (Nikon Metrology UK Ltd), Unit 5, Icknield Industrial Estate, \\ Tring, $\mathrm{UK}^{2}$ \\ E-mail: wenchao.cao@kuleuven.be
}

\begin{abstract}
.
A new beam hardening correction (BHC) method for multi-material industrial computed tomography (CT) is presented. Our method is a statistical learning approach of the Joseph and Spital (JS) segmentation framework. It consists of four steps. First, a multi-dimensional surface and its corresponding hyperplane are simulated based on the X-ray transmission model. They represent the polychromatic and monochromatic forward projection data. A beam hardening correction regression model is trained with the simulated data afterwards. Then the propagation length of the high X-ray absorbing materials is traced from an initial reconstruction. Finally, beam hardening is corrected with the regression model and the corrected image is reconstructed by filtered-back projection (FBP). The accuracy of this method is investigated and compared to that of the conventional JS implementation. Results indicate that the proposed method can achieve comparable performance to the conventional JS method with only $0.6 \%$ of its memory requirement. It is further evaluated on a four-material phantom simulated data set and two experimental dual-material data sets. Results prove that it can not only reduce the appearance of beam hardening, but also increase the dimensional measurement accuracy.
\end{abstract}

Keywords: algorithms, multi-material, beam hardening, dimensional metrology, X-ray $\mathrm{CT}$ 


\section{Introduction}

Beam hardening artefacts stem from the fact that the X-ray spectrum extends over a broad range of energies and the attenuation coefficients are energy-dependent [Bushberg and Boone, 2011]. Commonly used beam hardening correction (BHC) algorithms are linearization, dual-energy, and iterative reconstruction algorithms. Linearization is the simplest software correction method, which is often realised by a polynomial fit [Herman, 1979]. It fails with multi-material objects because essentially it is based on one-toone mappings between mono- and poly-energetic measurements. Dual-energy technique decomposes the image intensity into a linear combination of two basis functions, namely photoelectric effect and Compton scattering. The weight of each basis function can be solved by obtaining scanned data sets at two different X-ray spectra [Alvarez and Macovski, 1976, Fessler et al., 2002, Zhang et al., 2014]. Since the energy-dependent information is stored in the basis functions, a pseudo-monochromatic image can be produced. The requirement for a specific hardware configuration limits the application of dual-energy technique. Iterative algorithms achieve beam hardening reduction by implementing the energy-dependent information in the reconstruction model [De Man et al., 2001, Brabant et al., 2012, Van Slambrouck and Nuyts, 2012], but these usually only achieve a compromise between the computer capacity and algorithm performance.

Despite being limited to single-material objects, linearization is the most universally accepted BHC method for its straightforward implementation and fast execution. Linearization is available in many commercial CT software packages. Users sometimes do a partial correction for a multi-material object by applying ubiquitous linearization to different materials. Beam hardening caused by different materials are normally either under- or over-corrected. To achieve better results, linearization can be combined with segmentation. Different materials are segmented in the reconstructed volume following an initial reconstruction. Then linearization is applied to each material. The commonly used segmentation scenario is the JS method [Joseph and Spital, 1978] [Joseph and Ruth, 1997]. Firstly, an initial reconstruction is needed to segment the high X-ray absorbing (hard) material from the reconstructed volume. The initial reconstruction is often paired with a partial correction based on the BHC curve of one of the materials. The propagation length to each detector pixel of the hard material is acquired from ray-tracing afterwards [Joseph, 1982]. Then a pre-calculated lookup table (LUT) is applied to correct the low X-ray absorbing (soft) material, and some additional tuning parameters are used to compensate for the hard material. Values in the LUT are related to the materials from which the object is made. Essentially, they are determined by the attenuation of X-ray beams under different material thicknesses and energies. Proposed adaptions of the JS method can be found in [Herman and Trivedi, 1983, Hsieh et al., 2000, Van de Casteele et al., 2004, Gao et al., 2006, Kyriakou et al., 2010]. Another

type of segmentation approaches seeks to segment both the soft and hard materials [Nalcioglu and Lou, 1979, Meagher et al., 1990, Krumm et al., 2008]. In [Krumm et al., 2008], the propagation lengths of both the soft and hard materials are traced in the 
initial reconstructed image. Each pixel is aligned as a point in a $3 \mathrm{D}$ surface. The first two dimensions the space are the propagation lengths of both materials, and the third dimension is the logarithmic transmission value. The point clouds are then fitted to a curve. The corresponding monochromatic points can be placed on a plane. By reprojecting the curve onto the plane, beam hardening can be corrected. One problem of this kind of approaches is the incorrect segmentation of the soft material. Because the grey values of the soft material pixels are often similar to those of the bright beam hardening streaks, a lot of the background pixels are misidentified as soft material. The result of this kind of approaches is not satisfactory in many instances.

Comparing these two types of segmentation methods, the former suits a wide range of applications in industry. The hard material is easier to segment because it is less contaminated by beam hardening compared to the soft material. An initial linearization of the hard material is often enough to partially alleviate artefacts that affect the segmentation. To the contrary, the contrast resolution of the soft material is low. Its segmentation is often unsatisfactory.

Although the theory of the segmentation method is well developed, some technical obstacles remain in its implementation. Most researchers investigating this method have utilised LUT in the implementation. Essentially, LUT is a set of discrete and finite data points corresponding to the propagation lengths of a material. A substantial amount of propagation lengths needs to be sampled in order to yield a smooth representation of an object. Therefore, the application of the segmentation method is often restrained to small dual-material objects due to the limited computer memory.

In this paper, we propose a new segmentation method modified from the JS method to generate a smooth function for the discrete data points. We employ the virtual spectral and material information to produce a multi-dimensional hypersurface and its corresponding hyperplane. A polynomial BHC regression model is trained using the virtual information afterwards. By segmenting the high absorbing materials from the initial reconstruction, we then used the logarithmic transmission value and the propagation length of the high absorbing materials as the independent variables of the regression model to get the dependent variable, which in our case is the estimated corrected grey value. This method can significantly reduce the computer memory requirement of the original JS method. Hence it can be applied to objects of any size and number of materials.

This paper is organised as follows. Section 2 presents the JS method and our modification, together with a description of the experimental design. In Section 3, we present results that compare the uncorrected and corrected reconstruction with both the JS method and our modified method. Section 4 discusses the findings.

\section{Methods and materials}

This section begins by introducing the original JS segmentation method, and then covers our modification of its LUT based implementation. The phantoms of our experiments 
are presented alongside the performance quantification metrics that are captured from the phantom images. Finally, the specifications of the experiments are given.

\subsection{Original LUT based JS method}

This section assumes an object that is comprised of two materials. In the conventional FBP reconstruction algorithm, the X-ray spectrum is assumed to be monochromatic. The relationship between the transmitted X-ray beam intensity $I$ after passing through a single material and the incident beam intensity $I_{0}$ is therefore modelled as

$$
-\ln \left(\frac{I}{I_{0}}\right)=p_{m}=\int \mu_{H} d l_{H}+\int \mu_{S} d l_{S}
$$

where $p_{m}$ stands for the logarithmic monochromatic projection value, $\mu_{H}$ and $\mu_{S}$ are the attenuation coefficients of the hard and soft material, respectively; $l_{H}$ and $l_{S}$ are their corresponding propagation lengths. The attenuation coefficient is a function of the propagation length.

In practice, the logarithmic transmission is modelled as:

$$
\begin{aligned}
p_{p} & =-\ln \left(\frac{\int S(E) \exp \left(-\int \mu_{H}(x, y, E) d l_{H}-\int \mu_{S}(x, y, E) d l_{S}\right) d E}{\int S(E) d E}\right) \\
& =-\ln \left(\frac{\int S(E) \exp \left(-\int \mu_{H}(x, y, E) d l_{H}\right) d E}{\int S(E) d E}\right)-\ln \left(\frac{\int S^{\prime}(E) \exp \left(-\int \mu_{S}(x, y, E) d l_{S}\right) d E}{\int S^{\prime}(E) d E}\right) \\
& =p_{H}+p_{S}
\end{aligned}
$$

where,

$$
S^{\prime}(E)=S(E) \exp \left(-\int \mu_{H}(x, y, E) d l_{H}\right)
$$

In the above equations, $p_{p}$ is the logarithmic polychromatic projection value; $p_{H}$ and $p_{S}$ are the polychromatic contributions of the hard and soft materials.

As seen in Equation 2, despite the material distribution along an X-ray beam, $p_{p}$ only changes with the propagation length. The materials can be distributed in any order without changing the signal on the detector [Nalcioglu and Lou, 1979]. Therefore, we assume that the X-ray beam enters into a slab of the hard material and then enters into the soft material, which means the former can be seen as a filter of the latter. $S^{\prime}(E)$ is the 'hardened' spectrum by the hard material with propagation length $l_{H}$. Beam hardening artefacts result from simplifying the spectrum $S(E)$ into a single energy level in the FBP algorithm.

There are two stages of the JS method. The first stage involves building a LUT for the soft material correction. In Equation 2, $S^{\prime}(E)$ is a function of $l_{H}$. After discretizing the propagation length with a certain step, for each step a polynomial fit $f$ is used to linearize the polychromatic contribution of the soft material $\left(p_{S}\right)$ to the monochromatic contribution $\left(m_{S}\right)$ under the corresponding $S^{\prime}(E)$ (Equation 4$)$. 


$$
m_{S}=f\left(p_{s}\right)=a_{n} \times p_{s}^{n}+a_{n-1} \times p_{s}^{n-1}+\ldots+a_{0}
$$

For each discrete propagation length $l_{H}$ through the hard material, the LUT contains $4^{\text {th }}$ order polynomial coefficient for the soft material linearization. Because interpolation is needed to index values from the LUT, a small enough incremental step of $l_{H}$ is required to minimise the interpolation error. [Hammersberg and Mångård, 1998] suggested using a step size of $L_{H} / 1000$, where $L_{H}$ is the largest propagation length of the hard material.

One boundary condition should be imposed to Equation 4 is that, $a_{0}$ always equals 0 because it means there is no material.

The procedure for building up the LUT is as follows:

(i) The propagation lengths of all the hard materials are discretized. 1000 steps are used for each hard material. For example, if the largest propagation length of a hard material is $10 \mathrm{~mm}$, the step size for discretization is $0.01 \mathrm{~mm}$.

(ii) The hard materials are treated as filters for the soft material. All material propagation length combinations need to be considered as different combinations have different effects. Since the number of the discretized steps for each hard material is 1000 , the number of material combinations is $1000^{n}$, where $\mathrm{n}$ is the number of hard materials.

(iii) For each hard material combination, the corresponding polynomial coefficients for the soft material beam hardening correction are calculated. The polynomial order is 4 . Therefore, the total number of values in the LUT is $1000^{n} \times 4$.

In the second stage, after initial reconstruction, the hard material is segmented. The propagation length through the hard material corresponding to each pixel is traced. The monochromatic contribution of the hard material is calculated directly with:

$$
m_{H}=\mu_{H}\left(E_{0}\right) \times l_{H}
$$

$E_{0}$ is the monochromatic energy that the image is tuned at. The monochromatic contribution of the soft material is linearized by the polynomial coefficients in the LUT.

The main problem with this method is the memory requirement. In order for the algorithm to perform at a reasonable speed, the LUT needs to be in cache memory. The LUT size increase exponentially with the number of hard materials. Table 1 lists the memory requirements of the JS method with an increasing number of materials. For a typical high-end workstation, the L2 cache of the CPU is in the range of 8-15 MB, the memory requirement of the typical JS implementation exceeds this limit in Stage II with just three materials in the sample.

\subsection{Modified statistical learning approach}

The statistical learning approach presented in this paper is designed to reduce the memory requirement of the LUT method. 
In the polychromatic imaging, the relationship between the logarithmic image intensity and the propagation lengths of the materials is a non-linear surface in a $3 \mathrm{D}$ space. In the corresponding monochromatic image, this surface is simply a plane (Figure 2).

The first stage of the algorithm seeks a function to describe the relationship between the polychromatic surface and the monochromatic plane:

First a range of propagation lengths of the two materials $\left(l_{S}\right.$ and $\left.l_{H}\right)$ are sampled, so as to use Equations 1 and 2 to calculate the corresponding polychromatic and monochromatic projection values $(p$ and $m$ ). Taking the propagation length matrix of the hard material $\left(\mathbf{L}_{H}\right)$ and the polychromatic value matrix $(\mathbf{P})$ as the independent variable matrices, and the monochromatic value matrix $(\mathbf{M})$ as the dependent variable matrix, we can use them to fit a multivariate polynomial response surface function $\mathrm{f}\left(\mathbf{L}_{H}, \mathbf{P}\right)$ (Figure 1a). Notice that variables $p, l$, and $m$ are respectively elements of matrices $\mathbf{P}, \mathbf{L}$, and $\mathbf{M}$.

Because industrial objects often consist of dense materials, high-degree polynomials are needed to approximate the highly nonlinear relationship between the independent and dependent variables. We use a four-degree multivariate polynomial regression with interaction terms. The equations are as follows:

$$
\mathbf{M} \approx f\left(\mathbf{L}_{\mathbf{H}}, \mathbf{P}\right)=\Sigma_{i_{1}=0}^{4} \Sigma_{i_{2}=0}^{4} \beta_{i_{1} i_{2}} \mathbf{L}_{\mathbf{H}}{ }^{i_{1}} \mathbf{P}^{i_{2}}+\epsilon
$$

where $\epsilon$ is the negligible error caused by background noise. In its full expression with all the terms, the equation would look as follows:

$$
\begin{aligned}
f\left(\mathbf{L}_{\mathbf{H}}, \mathbf{P}\right) & =\beta_{00}+\beta_{01} \mathbf{P}+\beta_{02} \mathbf{P}^{2}+\beta_{03} \mathbf{P}^{3}+\beta_{04} \mathbf{P}^{4} \\
& +\beta_{10} \mathbf{L}_{\mathbf{H}}+\beta_{11} \mathbf{L}_{\mathbf{H}} \mathbf{P}+\beta_{12} \mathbf{L}_{\mathbf{H}} \mathbf{P}^{2}+\beta_{13} \mathbf{L}_{\mathbf{H}} \mathbf{P}^{3}+\beta_{14} \mathbf{L}_{\mathbf{H}} \mathbf{P}^{4} \\
& +\beta_{20} \mathbf{L}_{\mathbf{H}}{ }^{2}+\beta_{21} \mathbf{L}_{\mathbf{H}}{ }^{2} \mathbf{P}+\beta_{22} \mathbf{L}_{\mathbf{H}}{ }^{2} \mathbf{P}^{2}+\beta_{23} \mathbf{L}_{\mathbf{H}}{ }^{2} \mathbf{P}^{3}+\beta_{24} \mathbf{L}_{\mathbf{H}}{ }^{2} \mathbf{P}^{4} \\
& +\beta_{30} \mathbf{L}_{\mathbf{H}}{ }^{3}+\beta_{31} \mathbf{L}_{\mathbf{H}}{ }^{3} \mathbf{P}+\beta_{32} \mathbf{L}_{\mathbf{H}}{ }^{3} \mathbf{P}^{2}+\beta_{33} \mathbf{L}_{\mathbf{H}}{ }^{3} \mathbf{P}^{3}+\beta_{34} \mathbf{L}_{\mathbf{H}}{ }^{3} \mathbf{P}^{4} \\
& +\beta_{40} \mathbf{L}_{\mathbf{H}}{ }^{4}+\beta_{41} \mathbf{L}_{\mathbf{H}}{ }^{4} \mathbf{P}^{1}+\beta_{42} \mathbf{L}_{\mathbf{H}}{ }^{4} \mathbf{P}^{2}+\beta_{43} \mathbf{L}_{\mathbf{H}}{ }^{4} \mathbf{P}^{3}+\beta_{44} \mathbf{L}_{\mathbf{H}}{ }^{4} \mathbf{P}^{4}
\end{aligned}
$$

Similar to the LUT method, $\beta_{00}$ should always be 0 . Getting the value of the $\beta$ coefficients can be treated as a least squares problem. We can use numerical methods (e.g. QR factorisation with column pivoting [Ciarlet and Lions, 1990]) to get the solution. One concern of using numerical methods is the computational cost. For a least squares regression with $\mathrm{N}$ training examples and $\mathrm{C}$ features, the total complexity is $O\left(C^{2} N\right)$. Here, $O$ is the notation of the computational complexity. Therefore, when the computational cost exceeds the computer power, we can use iterative methods like gradient descent.

The second stage of the algorithm involves applying the function in a BHC task: 


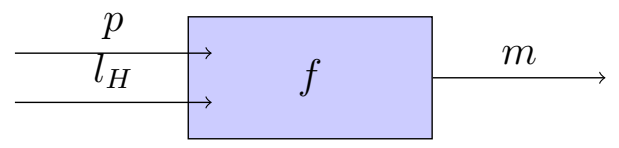

(a) Using pre-calculated variables $l_{H}, p$, and $m$ to fit the BHC model $f$.

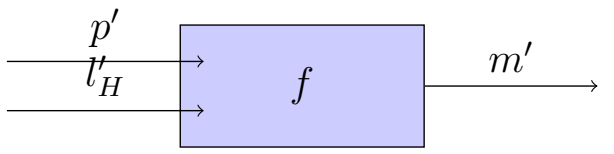

(b) Using variables $l_{H}^{\prime}$ and $p^{\prime}$ that are acquired from the scanning of an object to estimate the beam hardening corrected value $m^{\prime}$.

Figure 1: Data point calculation and model training.

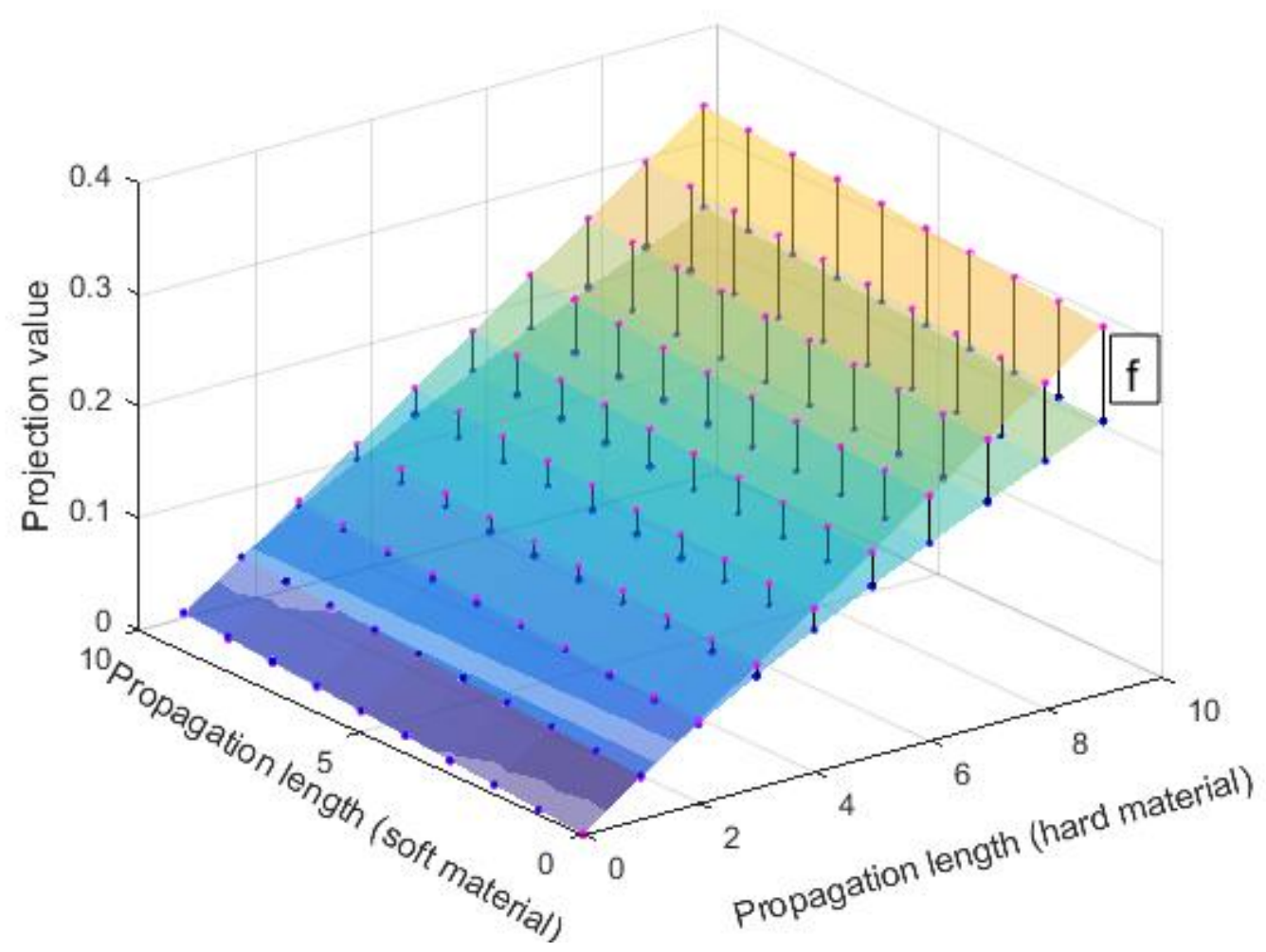

Figure 2: The 3D polychromatic curve and its corresponding 3D plane.

(i) The propagation length of the hard material is obtained from an initial reconstruction through ray-tracing.

(ii) Using the logarithmic transmission value $p^{\prime}$ of the projection image, and the propagation length $l_{H}^{\prime}$ to estimate the beam hardening corrected value $m^{\prime}$ (Figure 1b).

There are two advantages of using multivariate polynomial fit relative to LUT. In the first stage, the multivariate polynomial function can find the inherent relationship between the propagation length and the polychromatic image intensity with much fewer 
data points and yield a smooth curve as it is infinitely differentiable. The LUT method seeks to find the relationship between every pair of $p_{L}$ and $m_{L}$. Each line of the LUT can be seen as a distinct relationship determined by each individual $S^{\prime}(E)$, where $S^{\prime}(E)$ is shifted from $S(E)$ by $l_{H}$. The interval has to be small enough to allow a smooth interpolation. The multivariate polynomial function treats all the different combinations of the two materials as a whole imaging system under $S(E)$. For each material, an interval equals $L_{H} / 10$ is sufficient. The further advantage is reflected in the second stage. The first stage of the LUT method is memory efficient as it only requires one pair of $p_{S}$ and $m_{S}$. However, the memory requirement increases enormously in the correction stage. The modified method only requires saving the polynomial coefficients in the correction stage. The number of coefficients is $(n+1)^{k}$, where $\mathrm{n}$ is the polynomial order, and $\mathrm{k}$ is the input variable number. In practice, we can pre-calculate polynomial functions of several commonly used material combinations as the space complexity is low. A comparison of the memory requirements of the two methods for a limited range of the hard material propagation length $(0-10 \mathrm{~mm}$ with $0.01 \mathrm{~mm}$ step $)$ is given in Table 1. JS method exceeds L2 cache with three-material in Stage II of the algorithm. The proposed method is able to make use of the L1 cache up to four-material case thus offering significant advantages to the speed of the algorithm.

\begin{tabular}{c|c|c}
\hline Method & Stage I & Stage II \\
\hline LUT (two-material) & $8 \mathrm{kB}$ & $16 \mathrm{kB}$ \\
\hline LUT (three-material) & $8 \mathrm{kB}$ & $16 \mathrm{MB}$ \\
\hline LUT (four-material) & $8 \mathrm{kB}$ & $16 \mathrm{~GB}$ \\
\hline proposed (two-material) & $0.4 \mathrm{kB}$ & $0.1 \mathrm{kB}$ \\
\hline proposed (three-material) & $4 \mathrm{kB}$ & $0.5 \mathrm{kB}$ \\
\hline proposed (four-material) & $40 \mathrm{kB}$ & $2.5 \mathrm{kB}$ \\
\hline
\end{tabular}

Table 1: Memory requirements of the methods for a limited range of the hard material propagation length $(0-10 \mathrm{~mm})$.

\subsection{Phantoms}

Two phantom configurations are employed in this study. They are a multi-material rod based phantom and a multi-material hole cube (MM-HC).

The virtual design of the multi-material rod based phantom is comprised of three metal rods, two solid plastic rods, and one hollow plastic rod (Figure 3a). Details of the phantom geometry are given in Table 2. This virtual phantom configuration has been introduced in [Cao et al., 2018]. It is simulated in dual-material composition (results shown in Section 3.1.1) and four-material composition (results shown in Section 3.1.2).

The virtual phantom is manufactured in dual-material (stainless steel \& nylon). The rod arrangement is similar to that of the virtual phantom. There is no hollow nylon rod as it is difficult to manufacture. Figure $3 \mathrm{~b}$ shows a picture of the phantom. 


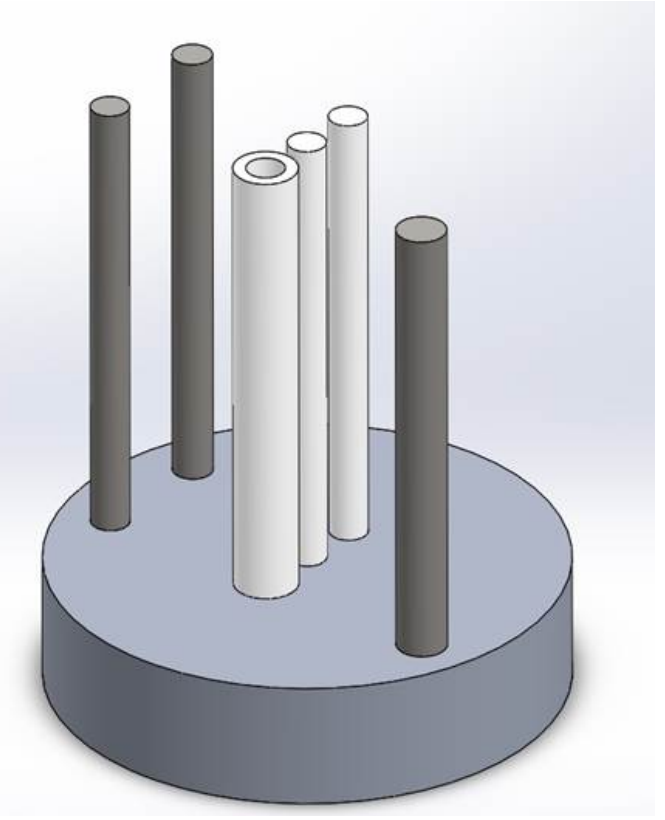

(a)

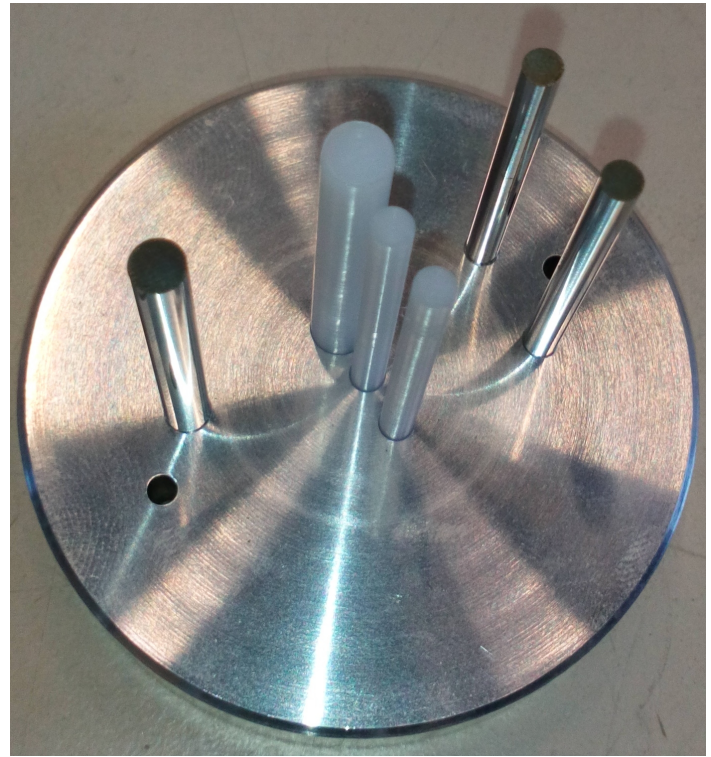

(b)

Figure 3: (a) A 3D drawing of the rod phantom. Metal is labeled in dark grey, and plastic is labeled in light grey. (b) A photo of the manufactured rod based phantom. The metal material is stainless steel, and the plastic material is nylon.

Since the precision of the dimension is not high, the scanning data set of this phantom is only used to quantify the artefact reduction in experimentation (results shown in Section 3.2.1).

The MM-HC phantom was developed by Physikalish-Technische Bundesanstalt (Braunschweig, Germany) for measurement testing. It is a dual-material (aluminium \& titanium) calibration cube which consists of 17 holes and 12 grooves (Figure 4). The phantom configuration has been introduced in [de Oliveira et al., 2017]. The radii of the 17 holes are measured at seven heights. The height is indicated by the groove location. The groove also serves to indicate the material where the measurement is

\begin{tabular}{|l|l|}
\hline Phantom element & Nominal radius $(\mathrm{mm})$ \\
\hline Metal $_{1}$ & 1.5 \\
\hline Metal $_{2}$ & 2.0 \\
\hline Metal $_{3}$ & 1.5 \\
\hline Plastic $_{1}$ & 1.5 \\
\hline Plastic $_{2}$ & 1.5 \\
\hline Plastic $_{3}$ - external & 2.5 \\
\hline Plastic $_{3}$ - internal & 1.5 \\
\hline
\end{tabular}

Table 2: Nominal radii of the rods. 
conducted. There are three kinds of groove locations: in the aluminium region; in the titanium region; in the dual-material region where the surrounding materials are partially aluminium and partially titanium. The nominal radius of each hole is $2 \mathrm{~mm}$. The CMM measured radii are shown in Table 3. For each hole, seven measurements at different heights (shown by the groove position, see Figure 4) are conducted. The scanning data set of this phantom is used to assess the algorithm performance in terms of dimensional measurement accuracy in experimentation (results shown in Section 3.2.2).

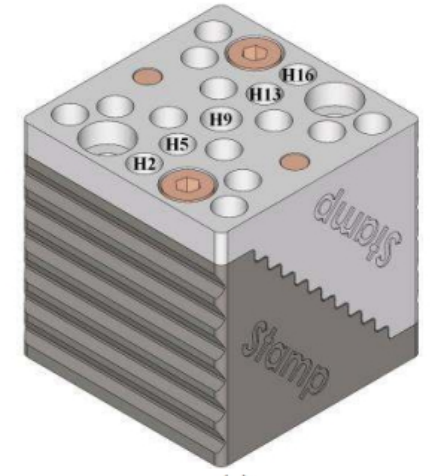

(a)

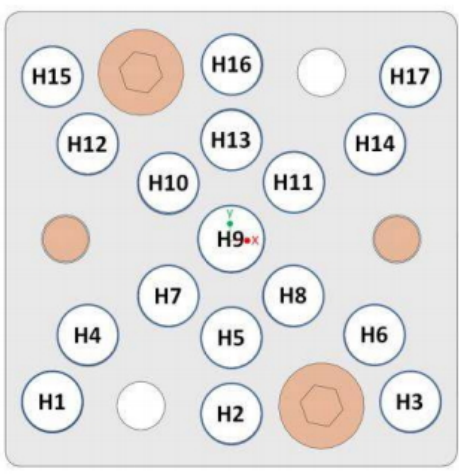

(b)

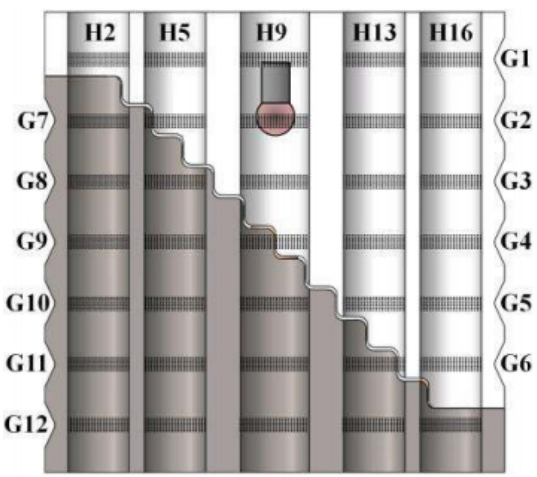

(c)

Figure 4: MM-HC design: (a) isometric view of the MM-HC; (b) top view of the design highlighting the position of the holes; (c) central cut of the design highlighting the stepwise diagonal cut separating materials, V-shaped grooves and calibration strategy, CMM probe (in red) added here for visualization of relative dimensions only [de Oliveira et al., 2017].

\subsection{Assessment metrics}

Three assessment metrics, two artefact indices and the dimensional offset, are applied to quantify the performance of the BHC algorithms. The artefact indices, which include streak index (SI) and cupping effect index (CI), are defined on the rod based phantom to quantify beam hardening artefacts. The dimensional offset is used to show the performance of the algorithms in regards to dimensional accuracy. The were introduced in [Cao et al., 2018].

The SI metric quantifies the grey value change caused by streaks between the rods, whereas the CI metric quantifies the grey value change caused by the cupping effects at the boundary of the rod. Both indices are calculated on line profiles of defined regions of interest (ROIs). The ROIs and line profiles are shown in Figure 5.

The following formulas are used in calculation: 


\begin{tabular}{|c|c|}
\hline Hole number & Reference radius $(\mathrm{mm})$ \\
\hline 1 & 2.00413 \\
\hline 2 & 2.00428 \\
\hline 3 & 2.00411 \\
\hline 4 & 2.00422 \\
\hline 5 & 2.00286 \\
\hline 6 & 2.00331 \\
\hline 7 & 2.00374 \\
\hline 8 & 2.00355 \\
\hline 9 & 2.25583 \\
\hline 10 & 2.00920 \\
\hline 11 & 2.00854 \\
\hline 12 & 2.01119 \\
\hline 13 & 2.01100 \\
\hline 14 & 2.01080 \\
\hline 15 & 2.01301 \\
\hline 16 & 2.01298 \\
\hline 17 & 2.01295 \\
\hline
\end{tabular}

Table 3: CMM measured radii of the holes. Each radius is averaged from seven measurements.

$$
\begin{aligned}
I_{a} & =\frac{I_{p 1}+I_{p 2}}{2} \\
I_{b} & =\frac{I_{v 1}+I_{v 2}}{2} \\
S I & =\frac{1}{m} \times \sum_{i=1}^{m} \frac{I_{a i}-I_{b i}}{\max \left(I_{a i}, I_{b i}\right)-M_{b}+C} \\
C I & =\frac{1}{n} \times \sum_{i=1}^{n} \frac{I_{a i}-I_{b i}}{\max \left(I_{a i}, I_{b i}\right)-M_{b}+C}
\end{aligned}
$$

where $I_{p 1}$ and $I_{p 2}$ are the two grey value peaks, and $I_{v 1}$ and $I_{v 2}$ are the two grey value valleys. $I_{a}$ and $I_{b}$ are the corresponding averages. Notice that the pixel indices of $I_{p 1}, I_{p 2}$, $I_{v 1}$ and $I_{v 2}$ are determined on the uncorrected images. Peak and valley image intensities on corrected images are defined as the image intensities corresponding to these same pixel indices. For CI calculation, since each line profile comprises only one valley, $I_{b}$ equals $I_{v} . m$ is the total number of line profiles in $\mathrm{ROI}_{S I}$, which equals $90 . n$ is the total number of line profiles in $\mathrm{ROI}_{C I}$ and it equals $180 . M_{b}$ is the average grey value of $\mathrm{ROI}_{b} . C$ is the maximum grey value of a 16-bit image, which equals 65535 .

The dimensional offset from the reference measurement is calculated by: 


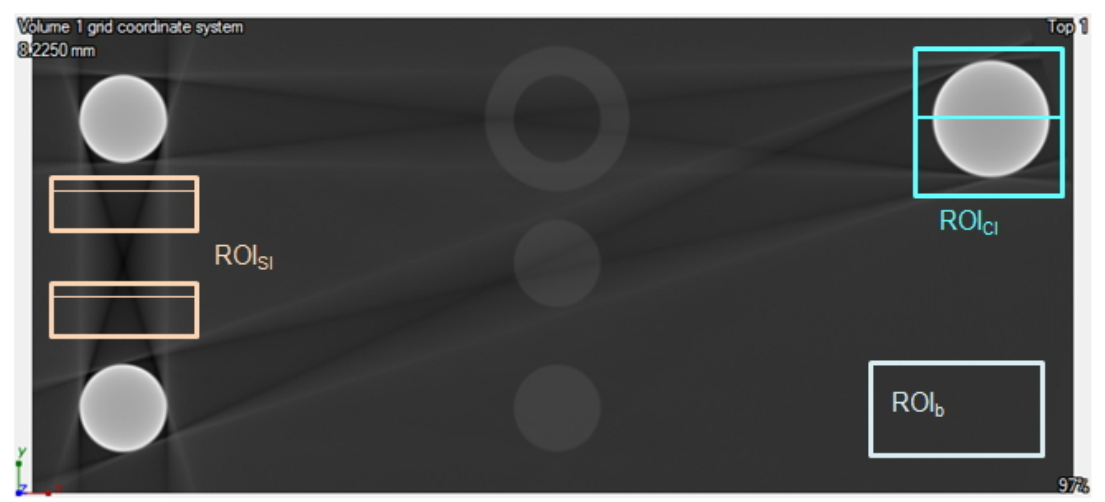

Figure 5: ROI definitions of the artefact indices. $\mathrm{ROI}_{S I}$ are the two rectangles for SI calculation. A total of 90 lines are drawn in $\mathrm{ROI}_{S I}$. Each rectangle is $45 \times 94$ pixels. For reason of clarity, only one is shown in each rectangle. On the rod in $\mathrm{ROI}_{C I}$, there are 180 line profiles in total, at $1^{\circ}$ angular step from each other. Each line extends $0.25 \mathrm{~mm}$ outside the rod in both directions.
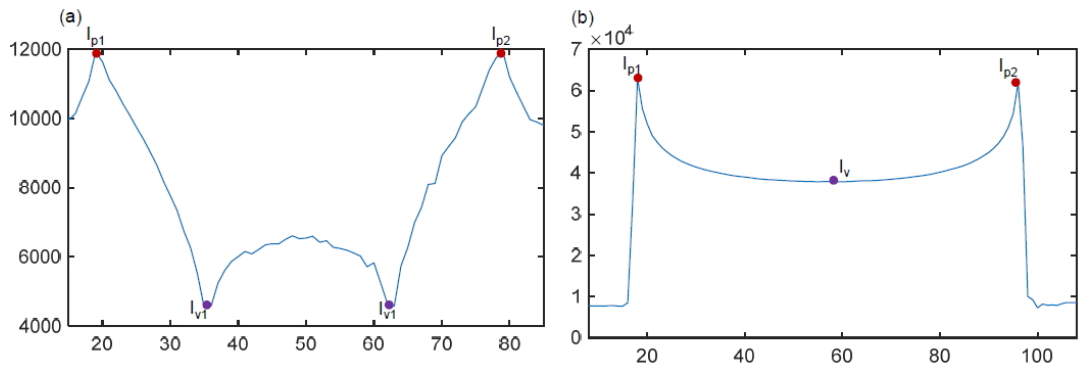

Figure 6: (a) An example SI line profile. The peaks and valleys correspond to the bright and dark streaks that the line traverses. (b) An example CI line profile. The peaks and valley correspond to the edge and centre of the rod that the line traverses.

Offset $(\mu \mathrm{m})=$ measured radius - reference radius

With simulated data, the corresponding monochromatic data set is used as reference. For the scanned data of the MM-HC phantom, the CMM measurement is used as reference.

The CT volume measurement is performed with VGStudio MAX 3.1 (Volume Graphics, Germany). The advanced mode with a search distance of $0.2 \mathrm{~mm}$ is used for edge detection. Each material is independently identified before edge detection.

\subsection{Data acquisition and processing}

The simulation was conducted with aRTist software [Bellon and Jaenisch, 2007]. The specifications of the simulation can be found in Table 4.

The rod based phantom was scanned under $240 \mathrm{kVp}$ without filter in a Nikon XTH450 system at KU Leuven. The spectral information used in the BHC algorithms was estimated with the transmission based method [Sidky et al., 2005]. The MM-HC 


\begin{tabular}{c|c}
\hline Source type & $\begin{array}{c}\text { reflection, } \\
\text { tungsten target }\end{array}$ \\
\hline Voltage range & $\begin{array}{c}\text { monochromatic: } 28 \mathrm{kV} \\
\text { polychromatic: } 150 \mathrm{kVp}\end{array}$ \\
\hline Focal spot & point \\
\hline Source to object distance & $250 \mathrm{~mm}$ \\
\hline Source to detector distance & $1000 \mathrm{~mm}$ \\
\hline Projection number & 3600 \\
\hline Detector & $0.2 \mathrm{~mm}$ pixel size \\
& $2000 \times 2000$ pixels \\
& 16 bit dynamic range \\
& energy-summing flat panel \\
\hline
\end{tabular}

Table 4: Specifications of the aRTist simulation.

phantom was scanned under $200 \mathrm{kVp}$ without filter in a Nikon XTH225 system at PTB. The spectral information used in the BHC algorithms was simulated with SpekCalc [Poludniowski et al., 2009].

FBP reconstructions were conducted with CTPro (X-Tek Systems Ltd., Tring, UK). The phantom element dimensions were measured in VGStudio MAX 3.1. The BHC methods were implemented in MATLAB R2015a on a laptop computer with 8GB RAM and a $2.5 \mathrm{GHz}$ quad-core processor. JS method and the modified method took respectively 1.5 hours and 30 mins to process a data set.

\section{Results}

\subsection{Simulation study}

The rod based phantom is used in the simulation study. The purpose of the first experiment is to validate the modified method by comparing it with the original JS method. A dual-material composition of the phantom is used. The second experiment is to further explore the application of the modified method in multi-material objects. A four-material composition is used. JS method is not used in the second experiment as the four-material object is beyond its capacity.

\subsubsection{Dual-material rod based phantom}

We validate the proposed algorithm by comparing with the JS algorithm with a dualmaterial rod based phantoms. The metal material is stainless steel, and the plastic material is polyphenylene sulphide (PPS). The spectrum used to generate the data set is a simulated $150 \mathrm{kVp}$ spectrum with $2 \mathrm{~mm}$ aluminium filter.

Figures $7 \mathrm{~b}$ and $7 \mathrm{c}$ illustrate the central slice of the reconstructed image corrected by the JS technique and the proposed modification. Both methods reduce a considerable 

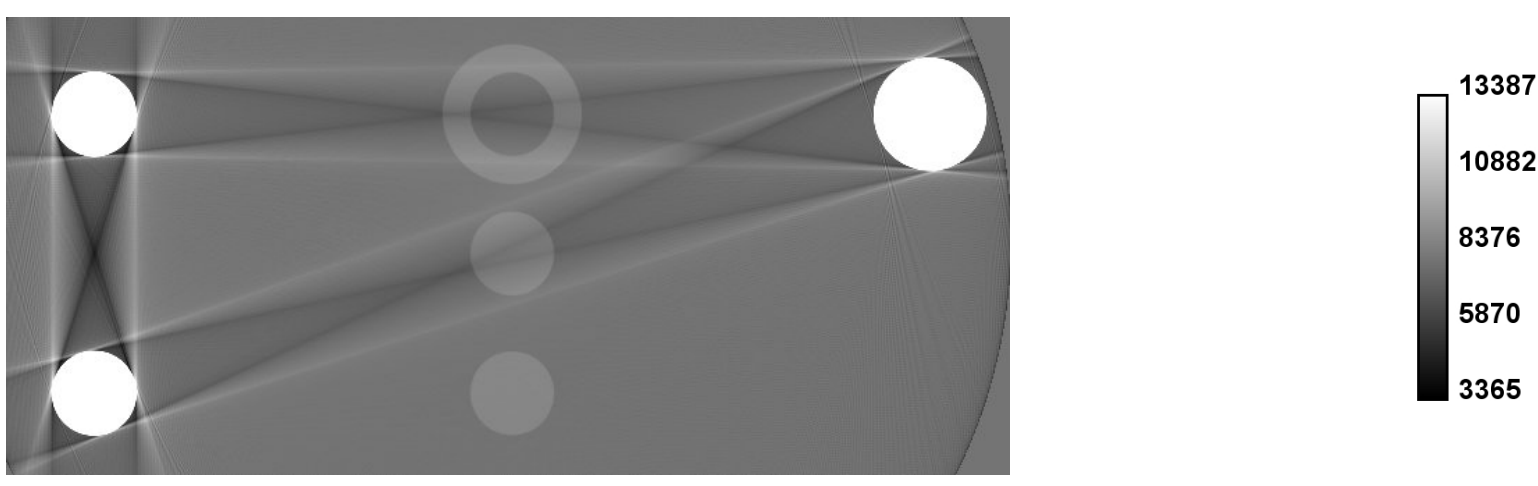

(a)

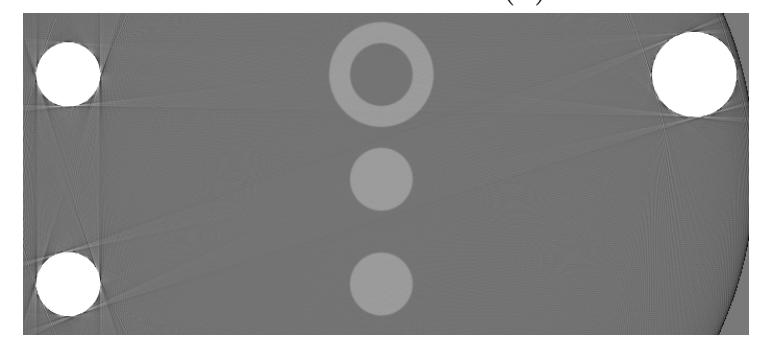

(b)

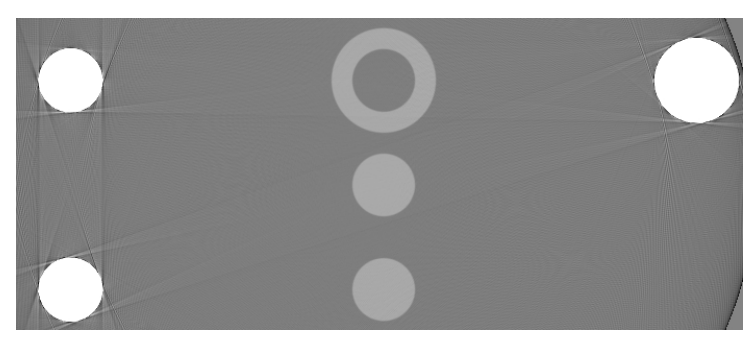

(c)

Figure 7: Reconstructed central slices of the simulated dual-material phantom. (a) Without correction. (b) With JS method. (c) With modified JS method.

amount of artefacts. The numerical result of the artefact reduction is given in Table 5. Both methods can significantly reduce the appearance of beam hardening. The performance is similar in this regard. Figure 8 shows the dimensional offset result. The dimensional offset is calculated as per Equation 12. The largest offset is $\sim 10 \mu \mathrm{m}$ without correction. Greater dimensional offsets are seen in the metal components than those of the plastic components. More improvement in dimensional accuracy can be observed in the metal components correspondingly after correction. Overall, the absolute value of the offset is under $3 \mu \mathrm{m}$. Our method outperformed the JS method by yielding smaller dimensional offsets in the metal rods.

\begin{tabular}{|l|l|l|}
\hline & SI & CI \\
\hline Polychromatic & 0.114 & 0.164 \\
\hline JS method & 0.005 & 0.003 \\
\hline Modified JS method & 0.004 & 0.005 \\
\hline Monochromatic & 0 & 0 \\
\hline
\end{tabular}

Table 5: SI \& CI values of the simulated dual-material phantom.

\subsubsection{Four-material rod based phantom}

We extend the proposed method to a four-material phantom consisting of one rod of each: titanium $\left(\mathrm{Metal}_{1}\right)$, copper $\left(\mathrm{Metal}_{2}\right)$, stainless steel $\left(\mathrm{Metal}_{3}\right)$. The spectrum used 


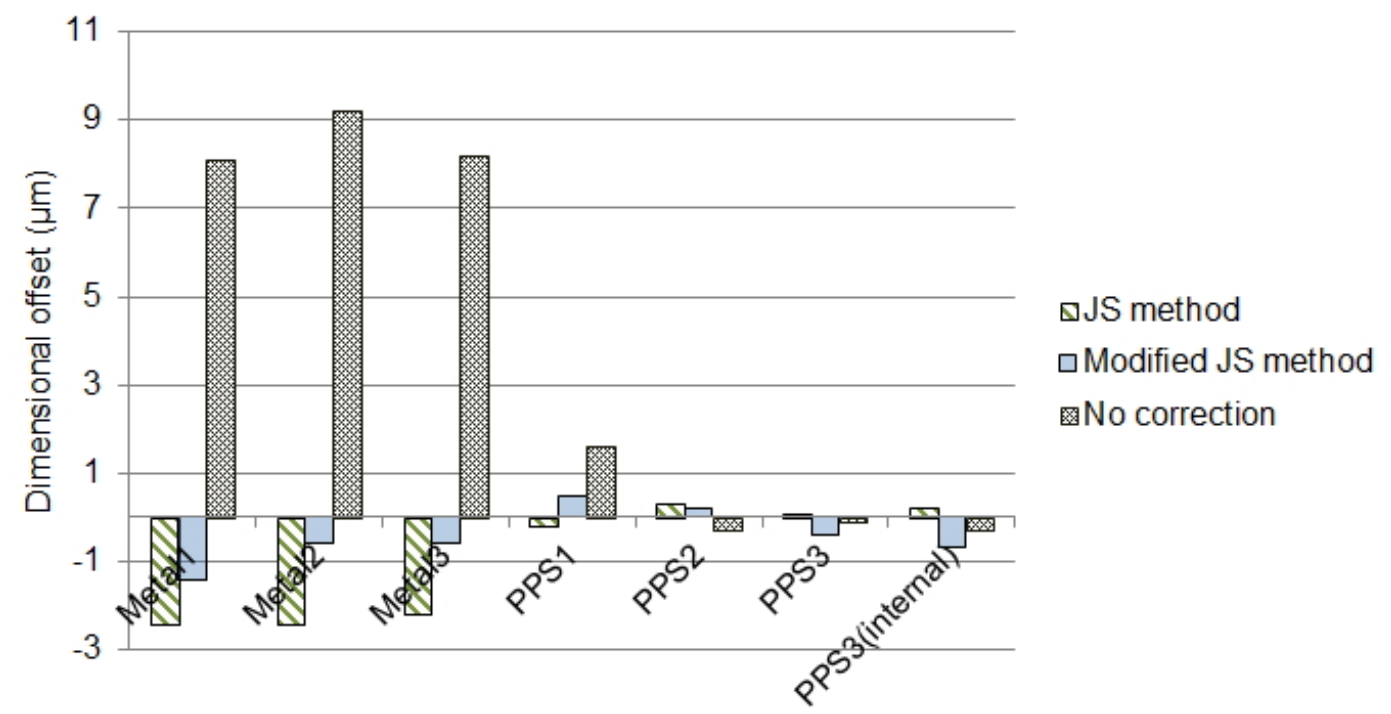

Figure 8: Dimensional offsets of the simulated dual-material phantom. The numbering of the steel rods are in accordance with the metal rods in Table 2, and the numbering of the PPS rods are in accordance with the plastic rods.

to simulate the data set is $150 \mathrm{kVp}$ with $2 \mathrm{~mm}$ aluminium filter. The expression for the four-material algorithm can be derived by extending Equation 6 to:

$$
\mathbf{M}=\sum_{i_{1}=0}^{4} \sum_{i_{2}=0}^{4} \sum_{i_{3}=0}^{4} \sum_{i_{4}=0}^{4} \beta_{i_{1} i_{2} i_{3} i_{4}} \mathbf{L}_{\mathbf{T}}{ }^{i_{1}} \mathbf{L}_{\mathbf{S S}}{ }^{i_{2}} \mathbf{L}_{\mathbf{C}}{ }^{i_{3}} \mathbf{P}^{i_{4}}+\epsilon
$$

where $\mathrm{L}_{T}, \mathrm{~L}_{C}, \mathrm{~L}_{S S}$ stand respectively for the propagation length matrices (described in Section 2.2) of titanium $\left(\mathrm{Metal}_{1}\right)$, copper $\left(\mathrm{Metal}_{2}\right)$, stainless steel $\left(\mathrm{Metal}_{3}\right)$. Figure $9 \mathrm{a}$ illustrates the reconstructed central slice of the image. Notice that the grey values of the image are in a broad range. The grey values of the streaks are at the low end, whereas that of the metal rods are at the high end. To better visualise the results, we presented the images in different brightness ranges. We can clearly see the three types of metals are of different brightness. The proposed method not only removes the cupping effects on the edges of the metal rods, but also retains the material differences.

Table 6 gives the numerical results of the metrics. There is a positive correction between CI and the metal density without correction. All artefact indices become negligible after correction. Figure 10 shows the dimensional measurement results. All the dimensional offsets are under $2 \mu \mathrm{m}$ in absolute value.

\begin{tabular}{|l|l|l|l|l|}
\hline & SI & CI $(\mathrm{Ti})$ & CI(steel) & CI(copper) \\
\hline Polychromatic & 0.093 & 0.064 & 0.134 & 0.249 \\
\hline Modified JS method & 0.009 & 0.005 & 0.005 & 0.007 \\
\hline Monochromatic & 0 & 0 & 0 & 0 \\
\hline
\end{tabular}

Table 6: SI \& CI values of the simulated four-material phantom. 


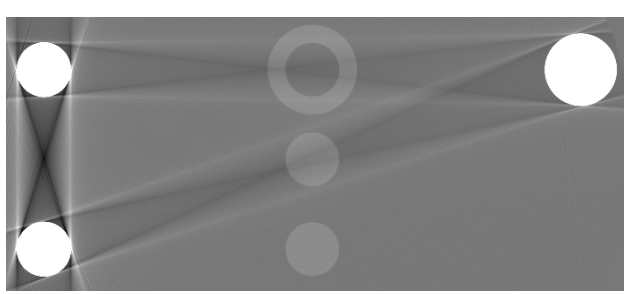

(a)

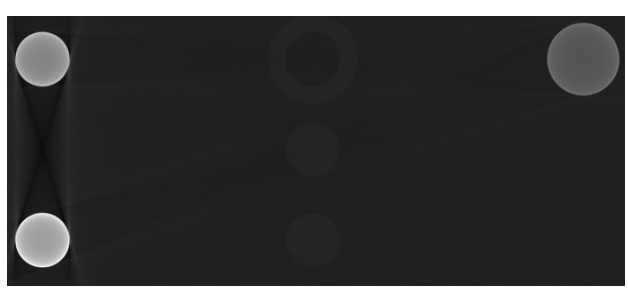

(c)

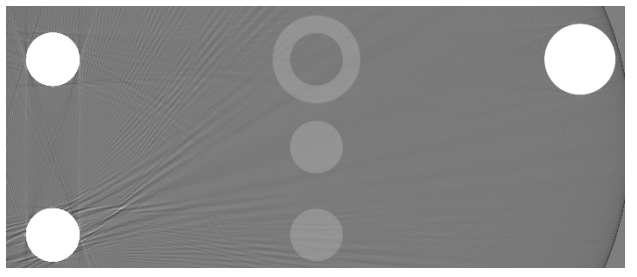

(b)

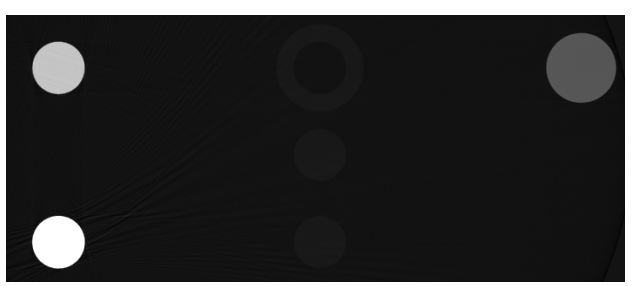

(d)
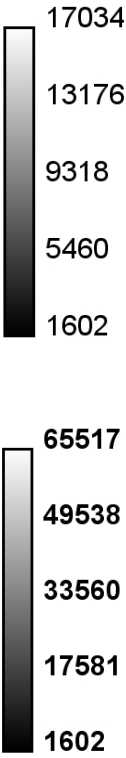

1602

Figure 9: Reconstructed central slices of the simulated four-material phantom. (a) \& (c): Without correction. (b) \& (d): With modified method. The first row is dedicated to showing the streaks, where we can see the proposed method has removed most of the streaks in the background. The second row is dedicated to showing the grey value differences of the metal materials.

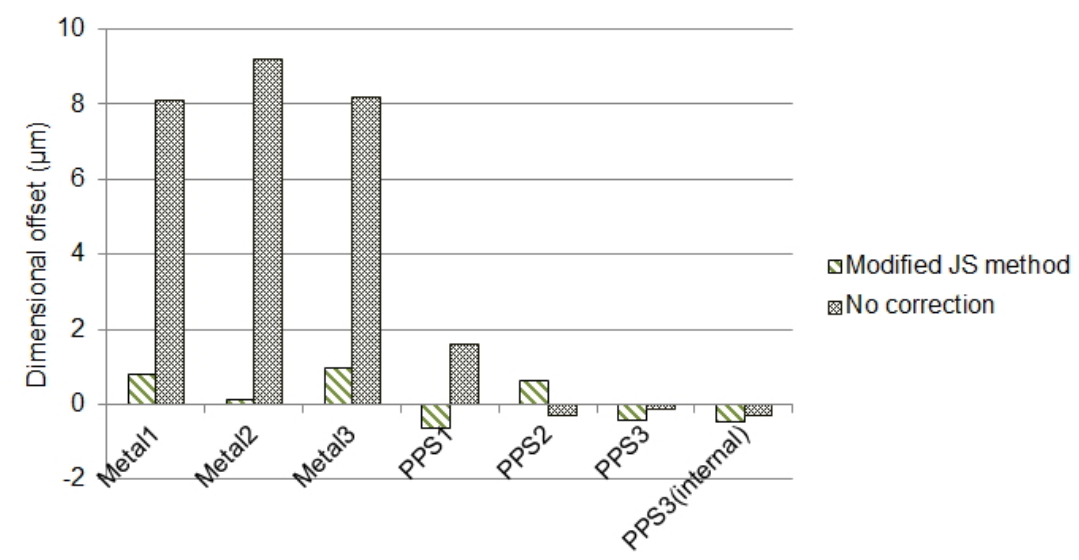

Figure 10: Dimensional offsets of the simulated four-material phantom.

\subsection{Experimental study}

The manufactured rod based steel-nylon phantom and the MM-HC are used for the experimental study. The results of the JS method and the modified method are presented side by side.

\subsubsection{Dual-material rod based phantom}

The rod based phantom is used to show visual quality improvement and artefact reduction. Figures 11 illustrates the reconstructed central slice without and with 


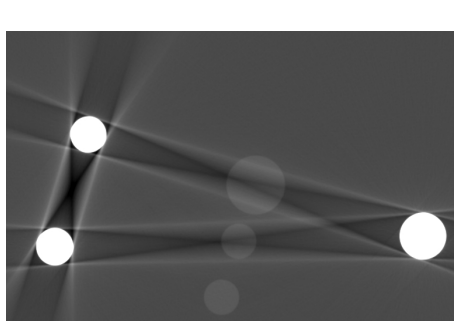

(a)

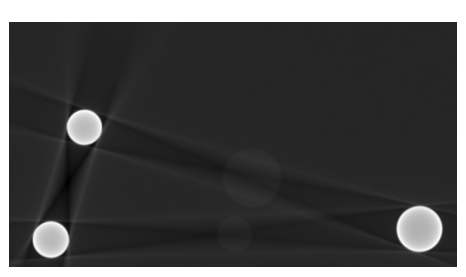

(d)

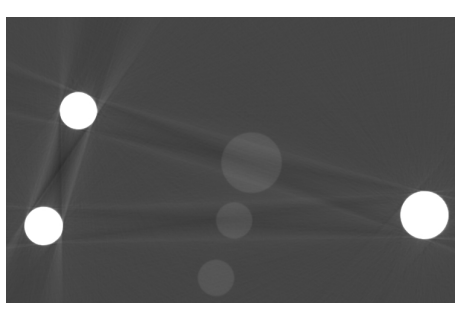

(b)

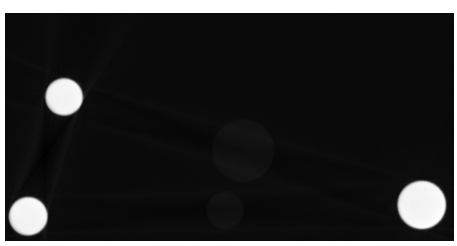

(e)

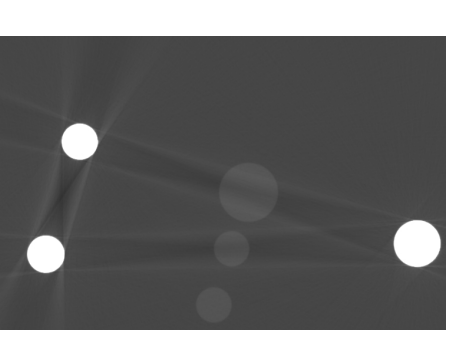

(c)

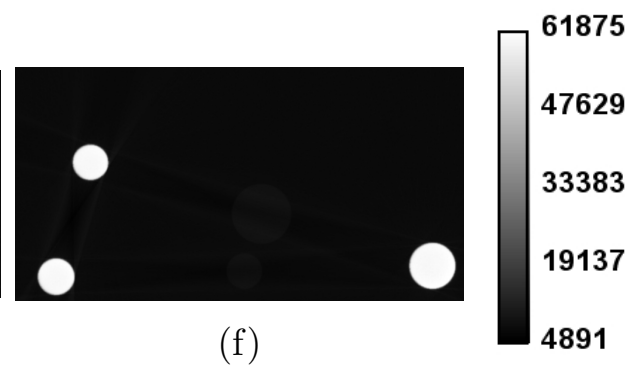

Figure 11: Reconstructed central slices of the manufactured dual-material phantom. (a) \& (d): Without correction. (b) \& (e): With JS method. (c) \& (f): With modified JS method.

correction at different brightness ranges. Both methods significantly reduce the appearances of beam hardening.

Table 7 lists the CI and SI to show the artefact reduction. Values of both indices are greater comparing to the indices in simulated phantom (Table 5), which is likely to be caused by a combined effect of beam hardening and scattering. After correction, the indices declined with both methods. CI appears to decrease more than SI. Nonetheless, both indices are greater than the values of simulation due to the remaining effect of scattering. Additional strategies are needed to reduce scattering, but it is beyond the scope of this paper.

\begin{tabular}{|l|l|l|}
\hline & SI & CI \\
\hline Polychromatic & 0.145 & 0.142 \\
\hline JS method & 0.033 & 0.009 \\
\hline Modified JS method & 0.030 & 0.009 \\
\hline
\end{tabular}

Table 7: SI \& CI values of the manufactured dual-material phantom.

\subsection{2. $M M-H C$ phantom}

The MM-HC phantom is used to show the dimensional measurement accuracy. Figures $12 \mathrm{a}$ and $12 \mathrm{c}$ show the reconstructed central slices from the top view. It is 
difficult to judge the image quality improvement as no obvious streaks or cupping effects can be observed in the central slice. However, it can be observed that the grey value difference between titanium and aluminium is degraded. As titanium is affected more by beam hardening, a horizontal line (the yellow line in Figure 12c) is drawn through the titanium part of the image. By comparing the grey value profile of the line (Figure 13), it is clear that the grey value that is degraded by beam hardening has been enhanced with correction.

The beam hardening reduction is further investigated by edge detection. When beam hardening presents, the grey value uniformity of a material is deteriorated in the reconstructed image. In the MM-HC phantom, the grey value of the titanium region should always be greater than the aluminium region as it is more attenuating. However, the grey values of many pixels in the aluminium region are close to titanium when beam hardening presents. Figures $12 \mathrm{~d}-12 \mathrm{i}$ show the edge detection of titanium from different views of the reconstructed image. The white granulated cells observed in the background and in the aluminium region are the misidentified material as a result of beam hardening. With correction, the material uniformity is increased. The misidentification is reduced accordingly. The algorithms show similar outcomes.

The absolute value of the dimensional offset is compared in Table 8. It presents the absolute value of the offset of each hole averaged from the measurements at different grooves. The grooves at the dual-material regions are excluded as their measurements introduce large deviation. Both algorithms can significantly improve dimensional measurement accuracy.

One interesting finding from the data analysis of the MM-HC phantom is the robustness of the algorithms with a simulated spectrum. As the true spectra of a CT system are often lacking, it is important that a BHC algorithm can achieve reliable performance with simulated spectra. 


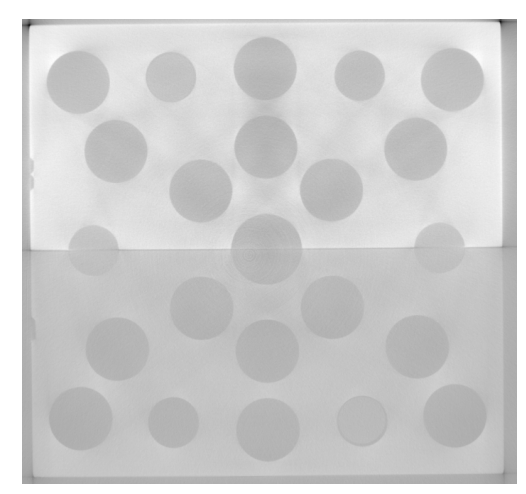

(a)

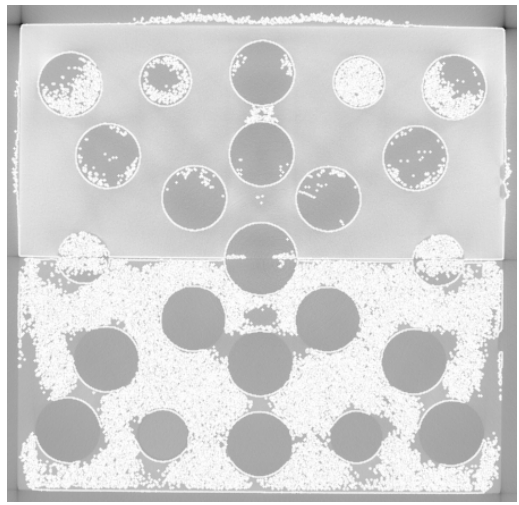

(d)

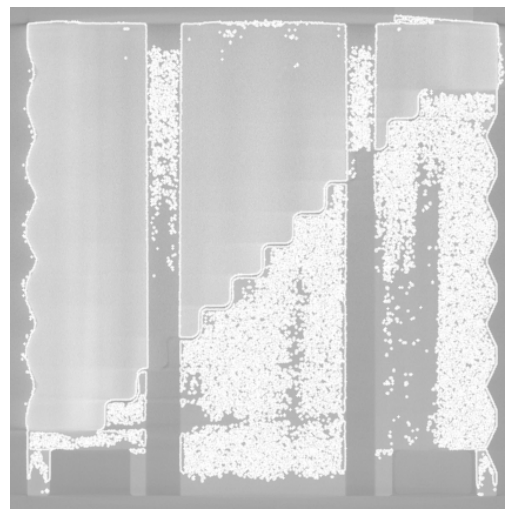

(g)

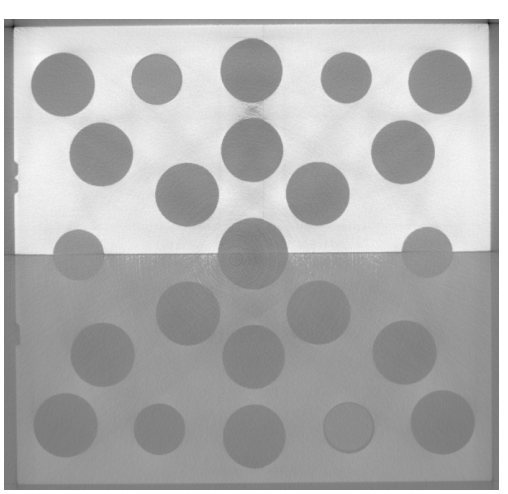

(b)

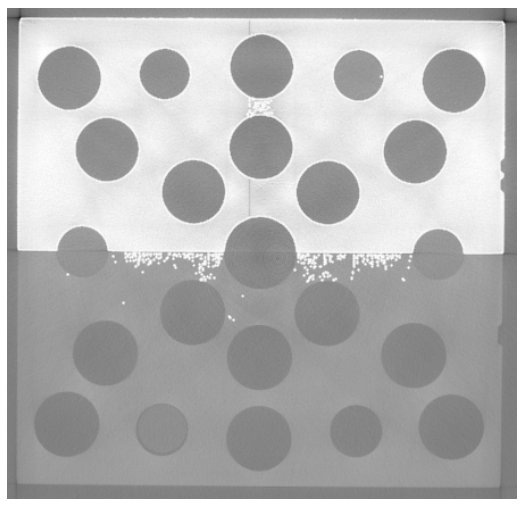

(e)

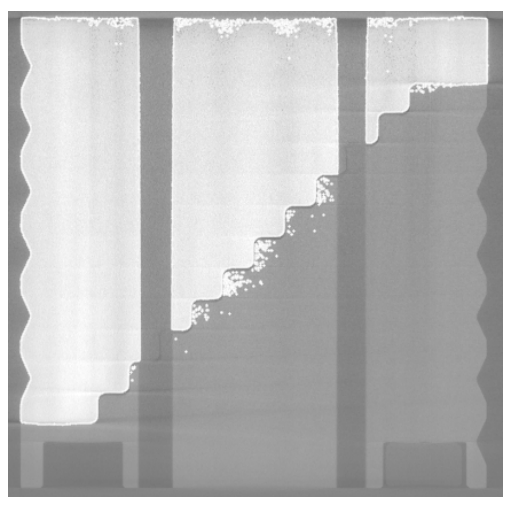

(h)

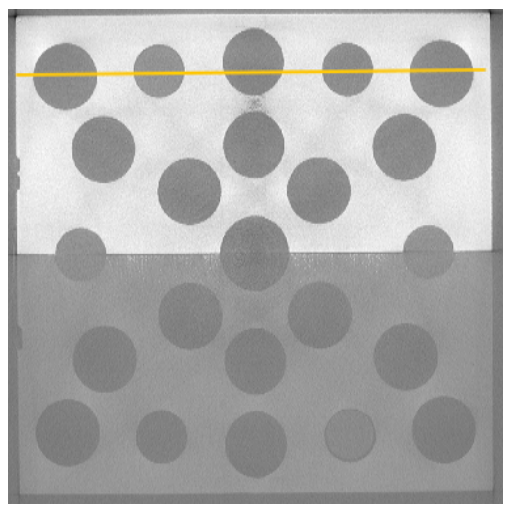

(c)

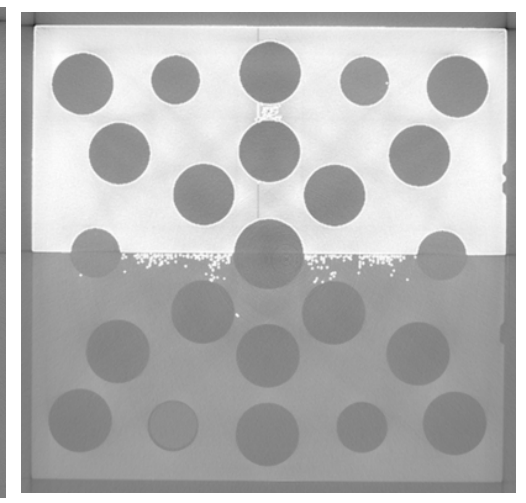

(f)

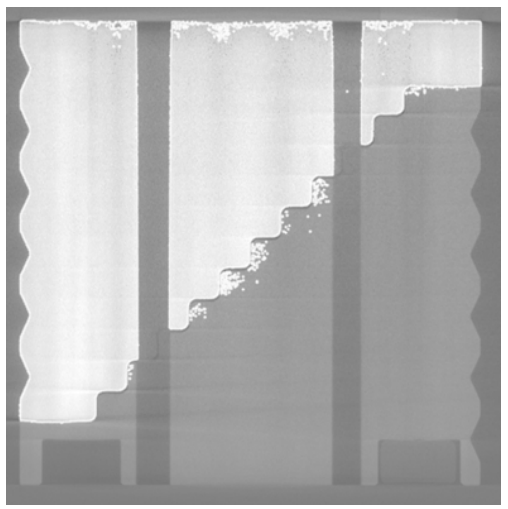

(i)

Figure 12: (a) - (c): Reconstructed central slices of the HH-MC phantom. (d) - (f): Edge detection of titanium (top view). (g) - (i) Edge detection of titanium (right view). 


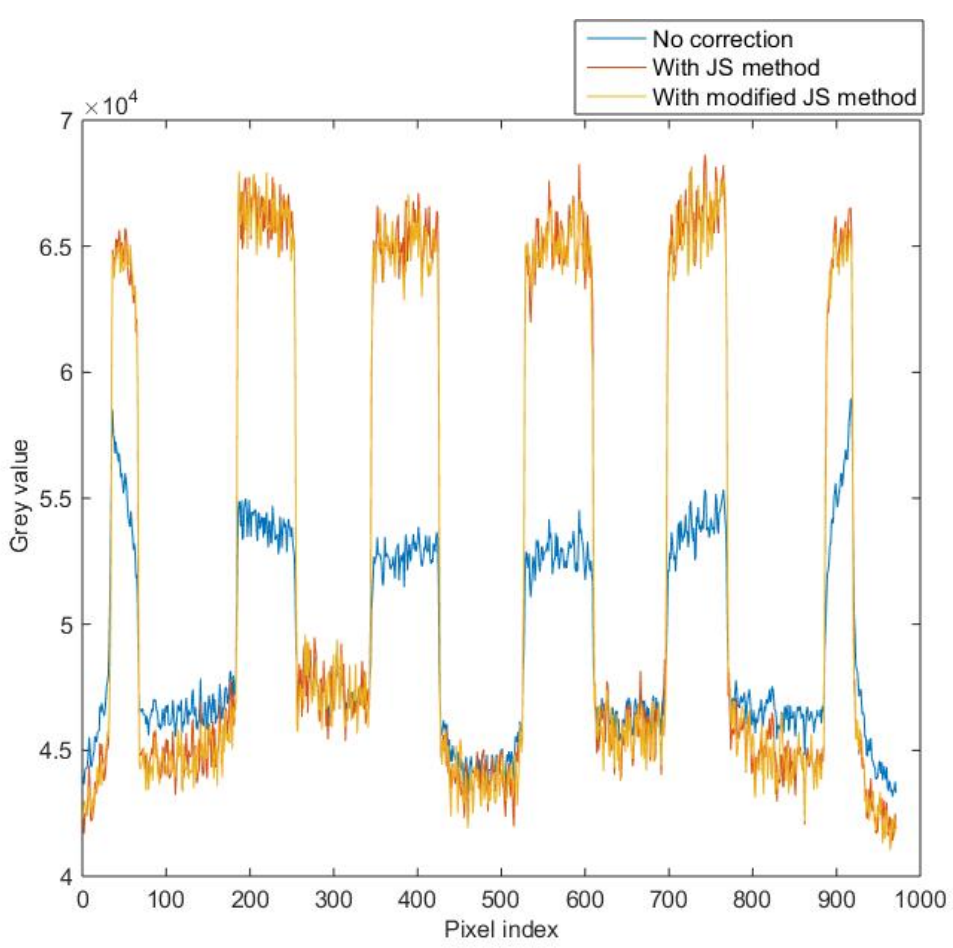

Figure 13: Grey value profile of a horizontal line through titanium presented in Figure 12(c).

\begin{tabular}{|c|c|c|c|}
\hline Hole number & No correction $(\mu \mathrm{m})$ & JS method $(\mu \mathrm{m})$ & Modified JS method $(\mu \mathrm{m})$ \\
\hline 1 & 59.83 & 0.67 & 0.59 \\
\hline 2 & 65.71 & 1.67 & 1.01 \\
\hline 3 & 41.74 & 1.01 & 1.01 \\
\hline 4 & 62.24 & 0.99 & 1.24 \\
\hline 5 & 2.43 & 1.02 & 2.04 \\
\hline 6 & 27.35 & 1.00 & 0.92 \\
\hline 7 & 28.06 & 1.04 & 0.73 \\
\hline 8 & 64.04 & 0.64 & 0.63 \\
\hline 9 & 19.92 & 1.58 & 1.39 \\
\hline 10 & 43.82 & 1.48 & 0.85 \\
\hline 11 & 11.85 & 1.02 & 1.1 \\
\hline 12 & 19.89 & 1.44 & 1.12 \\
\hline 13 & 1.62 & 1.16 & 1.13 \\
\hline 14 & 48.58 & 1.02 & 0.84 \\
\hline 15 & 23.6 & 0.42 & 0.66 \\
\hline 16 & 17.57 & 0.96 & 0.74 \\
\hline 17 & 38.65 & 0.98 & \\
\hline
\end{tabular}

Table 8: Averaged absolute value of the dimensional offset of the hole. 


\section{Conclusions}

We have introduced a non-iterative, segmentation based BHC method. Unlike most segmentation based methods which require LUT, our method directly uses the information acquired from the initial reconstruction to estimate the monochromatic value via a multivariate polynomial regression model.

We demonstrate the effectiveness of our method in multiple simulated and manufactured phantoms. Our method is capable of reducing the appearances of beam hardening, and improving the visual quality as well as dimensional measurement accuracy. Most of the results are compared with the original JS method. The results of our method are comparable to (and sometimes better than) those of the JS method. The result of the four-material phantom implies that our method can correct beam hardening in multi-material objects effectively. Because our method treats each material as an independent variable in a regression model, it is highly flexible with the number of materials. In addition, we train the regression model with simulated data. For each material combination, we only need to train the model once. Adding the number of materials does not increase much of the memory requirement. The only concern is the segmentation of different materials, as it requires repetitive manual work. Situations exist where one may want to sacrifice accuracy to some extent, in which we can group materials of similar densities (e.g. all the plastic materials) to achieve a partial correction.

The algorithm we present requires spectral knowledge, which can be measured or simulated. Section 3.2.2 shows reliable results with the simulated spectral information, indicating that our method is insensitive to spectral mismatch. This is of great importance as the true spectral information is often unavailable.

To sum up, there are mainly three advantages of our method compared to the JS method. First, our method can outperform JS method in a lot of applications. In addition, it can achieve reliable performance with significantly lower memory requirements. More importantly, it is parallelisable and fairly simple to implement in spite of the number of materials. It is conceivable that our method can no doubt be accelerated with an appropriate parallel implementation.

\section{Acknowledgements}

This work has received funding from the FWO-SBO MetroFleX project under grant agreement S004217N.

\section{References}

Jerrold T Bushberg and John M Boone. The essential physics of medical imaging. Lippincott Williams \& Wilkins, 2011.

Gabor T Herman. Correction for beam hardening in computed tomography. Physics in Medicine and Biology, 24(1):81, 1979. 
Robert E Alvarez and Albert Macovski. Energy-selective reconstructions in x-ray computerised tomography. Physics in medicine and biology, 21(5):733, 1976.

Jeffrey A Fessler, Idris Elbakri, Predrag Sukovic, and Neal H Clinthorne. Maximumlikelihood dual-energy tomographicimage reconstruction. In Proc DIR. spie, 2002.

Ruoqiao Zhang, Jean-Baptiste Thibault, Charles A Bouman, Ken D Sauer, and Jiang Hsieh. Model-based iterative reconstruction for dual-energy x-ray ct using a joint quadratic likelihood model. IEEE transactions on medical imaging, 33(1):117-134, 2014.

Bruno De Man, Johan Nuyts, Patrick Dupont, Guy Marchal, and Paul Suetens. An iterative maximum-likelihood polychromatic algorithm for ct. Medical Imaging, IEEE Transactions on, 20(10):999-1008, 2001.

Loes Brabant, Elin Pauwels, Manuel Dierick, Denis Van Loo, MA Boone, and Luc Van Hoorebeke. A novel beam hardening correction method requiring no prior knowledge, incorporated in an iterative reconstruction algorithm. $N d t \& E$ International, 51:68-73, 2012.

Katrien Van Slambrouck and Johan Nuyts. Metal artifact reduction in computed tomography using local models in an image block-iterative scheme. Medical physics, 39(11):7080-7093, 2012.

Peter M Joseph and Robin D Spital. A method for correcting bone induced artifacts in computed tomography scanners. Journal of computer assisted tomography, 2(1): 100-108, 1978.

Peter M Joseph and Christopher Ruth. A method for simultaneous correction of spectrum hardening artifacts in ct images containing both bone and iodine. Medical Physics, 24(10):1629-1634, 1997.

Peter M Joseph. An improved algorithm for reprojecting rays through pixel images. IEEE transactions on medical imaging, 1(3):192-196, 1982.

Gabor T Herman and Sushma S Trivedi. A comparative study of two postreconstruction beam hardening correction methods. Medical Imaging, IEEE Transactions on, 2(3): 128-135, 1983.

Jiang Hsieh, Robert C Molthen, Christopher A Dawson, and Roger H Johnson. An iterative approach to the beam hardening correction in cone beam ct. Medical physics, 27(1):23-29, 2000.

Elke Van de Casteele, Dirk Van Dyck, Jan Sijbers, and Erik Raman. A model-based correction method for beam hardening artefacts in x-ray microtomography. Journal of X-ray Science and Technology, 12(1):43-57, 2004.

Hewei Gao, Li Zhang, Zhiqiang Chen, Yuxiang Xing, and Shuanglei Li. Beam hardening correction for middle-energy industrial computerized tomography. Nuclear Science, IEEE Transactions on, 53(5):2796-2807, 2006.

Yiannis Kyriakou, Esther Meyer, Daniel Prell, and Marc Kachelrieß. Empirical beam hardening correction (ebhc) for ct. Medical physics, 37(10):5179-5187, 2010. 
O Nalcioglu and RY Lou. Post-reconstruction method for beam hardening in computerised tomography. Physics in medicine and biology, 24(2):330, 1979.

JM Meagher, CD Mote, and HB Skinner. Ct image correction for beam hardening using simulated projection data. IEEE Transactions on Nuclear Science, 37(4):1520-1524, 1990.

M Krumm, S Kasperl, and M Franz. Reducing non-linear artifacts of multi-material objects in industrial 3d computed tomography. Ndt \& E International, 41(4):242-251, 2008.

Peter Hammersberg and Måns Mångård. Correction for beam hardening artefacts in computerised tomography. Journal of X-ray Science and Technology, 8(1):75-93, 1998.

Philippe G Ciarlet and Jacques-Louis Lions. Handbook of numerical analysis, volume 8. Gulf Professional Publishing, 1990.

Wenchao Cao, Tao Sun, Greet Kerckhofs, Gemma Fardell, Ben Price, and Wim Dewulf. A simulation-based study on the influence of the x-ray spectrum on the performance of multi-material beam hardening correction algorithms. Measurement Science and Technology, 29(9):095002, 2018.

Fabricio Borges de Oliveira, Markus Bartscher, Ulrich Neuschaefer-Rube, Rainer Tutsch, and Hiller Jochen. Creating a multi-material length measurement error test for the acceptance testing of dimensional computed tomography systems. In 7 th Conference on Industrial Computed Tomography (iCT 2017), 2017.

Carsten Bellon and Gerd-Rüdiger Jaenisch. artist-analytical rt inspection simulation tool. In Proc DIR, pages 25-27, 2007.

Emil Y Sidky, Lifeng Yu, Xiaochuan Pan, Yu Zou, and Michael Vannier. A robust method of X-ray source spectrum estimation from transmission measurements: Demonstrated on computer simulated, scatter-free transmission data. Journal of applied physics, 97(12):124701, 2005.

G Poludniowski, G Landry, F DeBlois, PM Evans, and F Verhaegen. Spekcalc: a program to calculate photon spectra from tungsten anode x-ray tubes. Physics in medicine and biology, 54(19):N433, 2009. 